2021,

https://doi.org/10.1109//GARSS47720.2021.9554044

https://archimer.ifremer.fr/doc/00693/80508/

\title{
Oceanic Circulation in the Strait of Gibraltar revealed by AIS data Information
}

\author{
Le Goff C ${ }^{1,}{ }^{*}$, Mironov As ${ }^{1}$, Boussidi B ${ }^{1}$, Bordois L ${ }^{2,}{ }^{*}$, Dumas F ${ }^{2}$, Chapron Bertrand ${ }^{3}$
}

${ }^{1}$ e-odyn, Brest, France

2 SHOM, Brest, France

${ }^{3}$ Laboratory for Ocean Physics and Satellite Remote Sensing, Ifremer Brest, France

* Corresponding authors : C. Le Goff, email address : clement.legoff@e-odyn.com ; L. Bordois, email address : lucie.bordois@shom.fr

\begin{abstract}
:
Our understanding of ocean circulation to date still bears many unanswered questions. Despite the development of a variety of methods for observing upper ocean current velocity at different scales, our knowledge of this subject has yet to be significantly improved. Historically, the drift of vessels led to the discovery of the oceanic surface current. The Automatic Identification System (AIS), originally intended to avoid collision in maritime traffic, contains the necessary information to retrieve vessel drift due to the surface current. Thus, the AIS data can be processed to derive sea-surface current components for any region in which there is maritime traffic. A dedicated inversion scheme has recently been theoretically formulated and implemented. In this study, we report the preliminary results obtained during an experimental campaign conducted aboard the oceanographic vessel Atalante, in the strait of Gibraltar, in October 2020. A map of oceanic circulation was calculated using the drift of all vessels emitting AIS messages within the strait and validation was achieved with the help of an Acoustic Doppler current profiler (ADCP) aboard the Atalante.
\end{abstract}

Keywords : AIS, Oceanic Surface Current, Drift speed, Tides 


\section{INTRODUCTION}

The measurement, understanding and modelling of ocean current structure still remains an open topic for investigation in oceanography. Many efforts have been made to offer a robust approach to estimating upper ocean current velocities at different spatio-temporal scales. The Automatic Identification System (AIS) dedicated to collision avoidance for maritime traffic contains information related to vessel drift due to the surface current. As explained below, the combined analysis of AIS messages from different vessels in a given location allows for the estimation of current velocity components in that region. Recently introduced, this approach has been implemented by eOdyn in different regions around the globe and is the subject of validation for various sea-state and meteorological conditions.

The Strait of Gibraltar is the only connection between the Mediterranean Sea and the Atlantic Ocean. This geographical region is characterised by a complex oceanic current structure conditioned by a bottom topography that includes various sills and the constant deficit of the Mediterranean Sea due to evaporation, as well as the predominance of the semi-diurnal and diurnal tide flow. All of these make this a key spot for the study of oceanic circulation [1].
In this work we report the preliminary findings from an experimental campaign conducted aboard the oceanographic vessel Atalante in the Gibraltar strait area, in October 2020. This campaign was dedicated to combining different instrumental and methodological approaches for measuring the upper ocean current velocity. Our main objective was to validate the analysis of AIS messages generated by maritime traffic in the region, as a method for sea-surface current estimation.

\section{Data And Methods}

\section{A. Experimental Campaign}

The experimental campaign, Protevs Gibraltar, was conducted aboard the oceanographic vessel Atalante between the $3^{r d}$ and $23^{r d}$ of October 2020 . The systematic recording of AIS data messages from all vessels navigating the strait, as illustrated in figure 1 (a), allows for the calculation of each vessel's drift. The same idea was developed by Richardson [2] before the systematic use of AIS data messages. Given that a significant proportion of maritime traffic is composed of vessels which have an underwater area equivalent to that above water, the leeway-drift due to the lateral wind effect is negligible [3]. Under this assumption, vessel drift is then mostly due to the oceanic surface current. In this study particular attention was paid to the region around the Camarinal Sill, figure 1 (b), and the divergence of the current fields.

\section{B. Vessel Drift}

Aboard the Atalante, vessel drift is characterised by magnitude and direction. The vocabulary and a diagram are presented in figure 2. When projected onto the zonal and meridional axes, the drift can be written as follows:

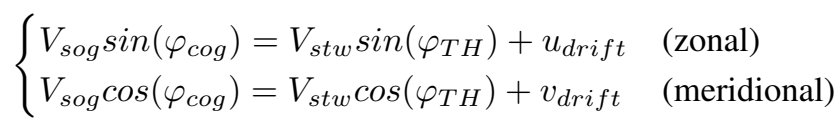

where $V_{\text {sog }}$ is the speed over ground, $\varphi_{\operatorname{cog}}$ the course over ground, $V_{s t w}$ the speed through water and $\varphi_{T H}$ the true heading of the Atalante vessel. Among these variables, all, except the $V_{s t w}$, are present in the AIS data messages which are emitted by vessels at high frequency intervals $(\approx 10 \mathrm{~s})$. A hull-mounted ADCP measured the velocity of the upper ocean currents below the Atalante. Along a transect within the strait, at a speed of $\approx 8 \mathrm{kn}$, comparisons were drawn 

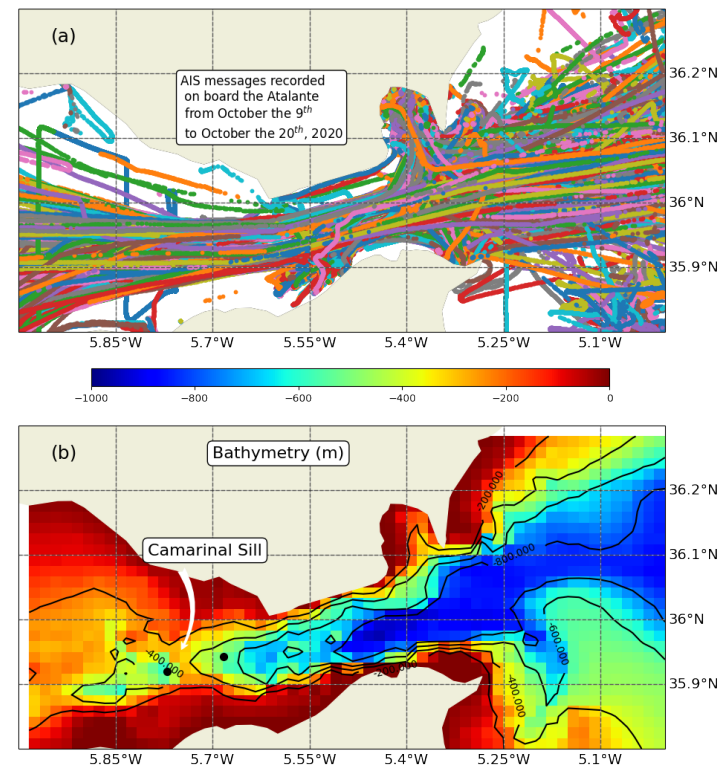

Fig. 1. (a) AIS messages recorded during the period of the experimental campaign onboard the Atalante. (b) Bathymetry of the Strait of Gibraltar.

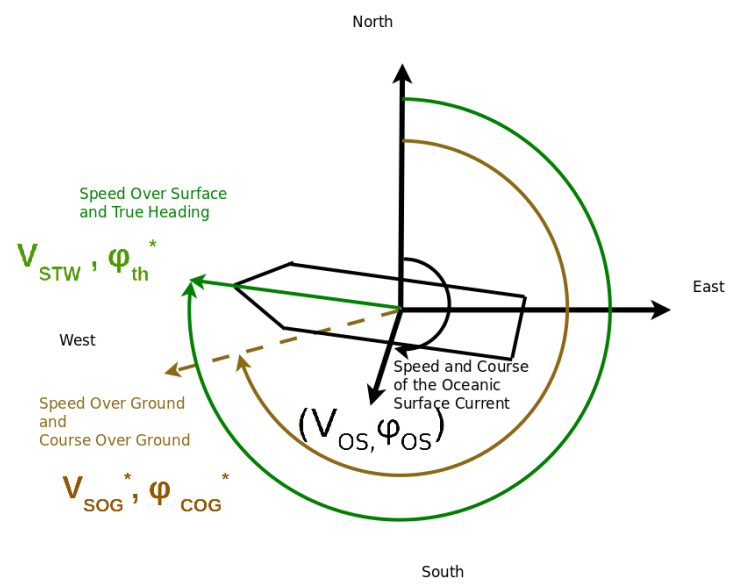

Fig. 2. Measurement diagram and definitions. Variables with an asterisk are known and transmitted via the AIS messages. The other variables are calculated. When projected over the zonal and meridional axes $V_{d r i f t}$ (or $V_{o s}$ when assimilated to the oceanic surface current) and $\varphi_{\text {drift }}$ or $\varphi_{o s}$ become $u_{d r i f t}$ and $v_{d r i f t}$.

between the zonal and meridional velocities measured by the $\mathrm{ADCP}$ and the zonal and meridional drift components. Results are presented in figure 3. A positive correlation $r^{2} \approx 0.8$ and $R M S E \approx 14 \mathrm{~cm}$ can be observed for the zonal and meridional components. Regarding the hull-mounted ADCP, we used the estimates at $25 \mathrm{~m}$ because at shallower depths the results are affected by the motion of the vessel. Considering this result we can assume that the drift is essentially due to the oceanic current. The drift velocities considered $u_{d r i f t}$ and $v_{\text {drift }}$ become the oceanic surface current $u_{o s}$ and $v_{o s}$. Applying this technique to all vessels navigating in the strait is not so straightforward given that the speed through water is not transmitted in the AIS messages.
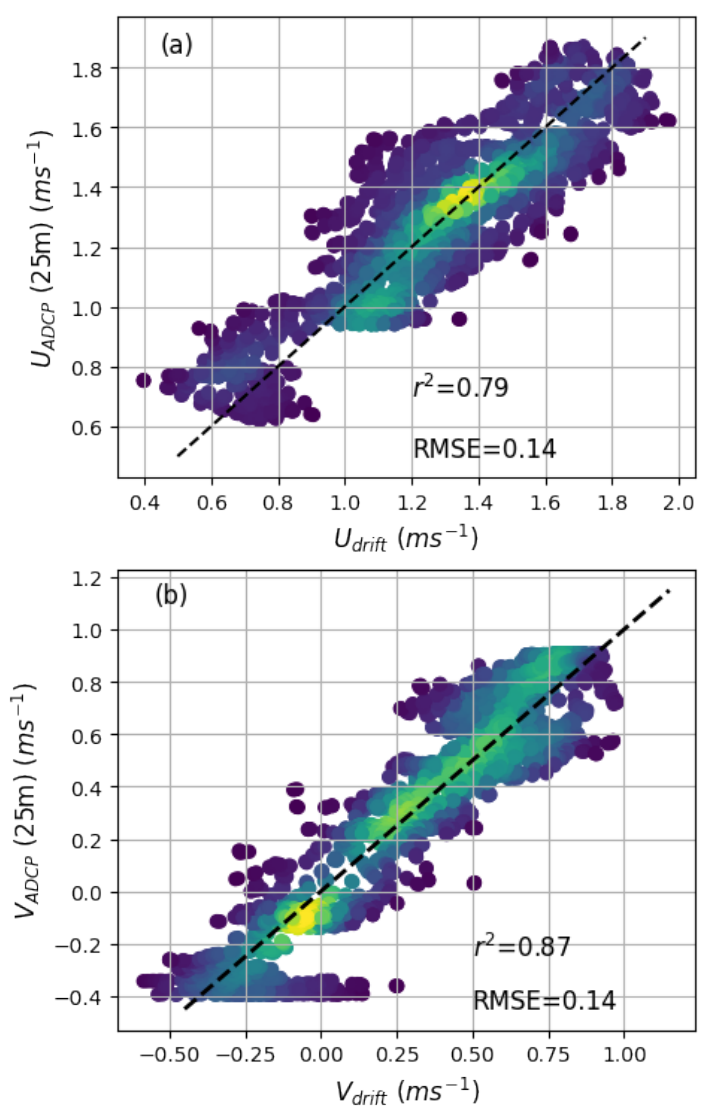

Fig. 3. Comparisons between the drift and the oceanic current obtained from the hull-mounted ADCP at $25 \mathrm{~m}$ for the zonal (a) and the meridional (b) components

\section{Reconstruction of Vessel Speed Through Water}

In this part we considered the vessels collectively rather than individually. Vessels and their associated AIS messages were considered to be in homogeneous surface conditions in both space and time. Given this, the AIS messages were aggregated within a spatio-temporal box of $1 / 24^{\circ}$ and one hour. For each AIS message, we have two equations and three unknowns (the magnitude of $V_{s t w}$ and the two components of the current). For $n$ AIS messages, we therefore have $2 n$ equations. However, using the space-time homogeneity assumption, this leaves only $n+2$ unknowns to be solved. The following system is thus considered:

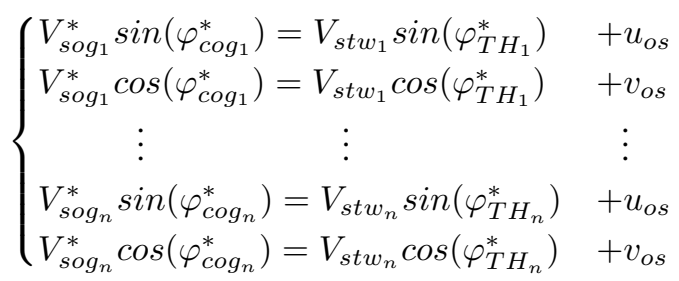

The variables with an asterisk are transmitted by the AIS messages and the speed though water $V_{s t w_{n}}$ had to be calculated. Once completed, we collected the estimates of the speed through water for each vessel and rearranged them along their trajectories. 
Figure 4 shows the speed over ground (green), and the estimate of the speed through water (blue; as obtained from solving the system of equations 2) for a randomly selected vessel navigating on October $10^{\text {th }}, 2020$. Regarding the timeseries of the speed though water, we filtered our results using the Empirical Mode Decomposition (EMD) and kept only the trend obtained [4]). From this filtered estimate of speed through water, we then used the set of equations 1 and calculated the zonal and meridional components of the oceanic surface current as follows:

$$
\left\{\begin{array}{l}
u_{o s}=\mathcal{F}\left(V_{\text {stw }}\right) \sin \left(\varphi_{T H}\right)-V_{\text {sog }} \sin \left(\varphi_{\text {cog }}\right) \\
v_{\text {os }}=\mathcal{F}\left(V_{\text {stw }}\right) \cos \left(\varphi_{T H}\right)-V_{\text {sog }} \cos \left(\varphi_{\text {cog }}\right)
\end{array}\right.
$$

where $\mathcal{F}\left(V_{s t w}\right)$ represents the filter for the speed through water. Results for the zonal and meridional components of the oceanic surface current are shown in blue and orange in figure 4 (b).

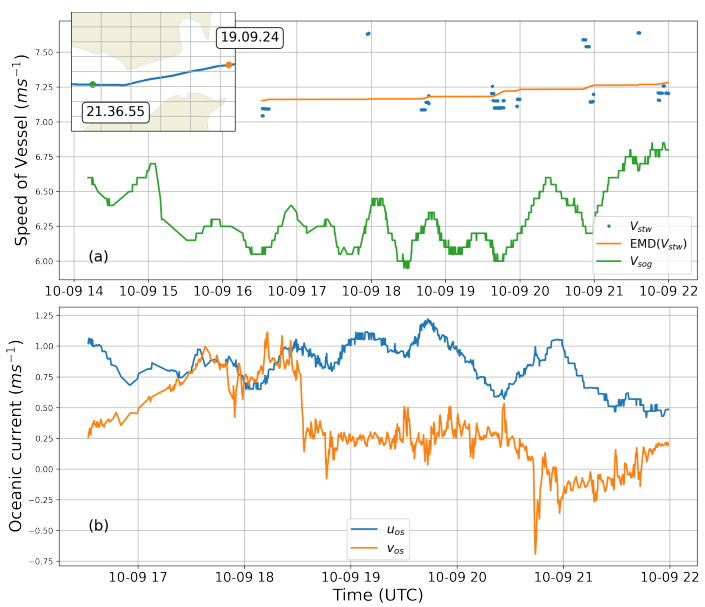

Fig. 4. (a) Top left: Trajectory of a randomly selected vessel navigating on October $10^{t h}, 2020$. In green, the speed over ground, in blue the reconstructed speed through water and in orange the result obtained after filtering using the Empirical Mode Decomposition (EMD). (b) Reconstructed oceanic surface current velocity obtained from the set of equations 1 with the speed though water obtained from the EMD

\section{Optimal Interpolation}

Once data sets had been obtained for both the zonal and meridional velocity components, we applied a classic optimal interpolation in order to smooth and map the results in both space and time [5]. Optimal interpolation is a mathematical tool used to merge background information $\left(x^{b}\right)$ from a priori knowledge, in this case, the average over time for the zonal and meridional current estimates and observations $(y)$. The result of the optimal interpolation is Gaussian with a mean $x^{s}$ and a covariance $P^{s}$ given by:

$$
\begin{aligned}
x^{s} & =x^{b}+K\left(y-H x^{b}\right), \\
P^{s} & =B-K H B,
\end{aligned}
$$

with the gain $K=B H^{\top}\left(H B H^{\top}+R\right)^{-1}$,

where $H$ is the observation operator, thus creating the link between the observation and the background. Here, $H$ is the identity matrix.

\section{E. Helmholtz-Hodge Decomposition}

Once the interpolated field obtained, we applied a Helmholtz-Hodges Decomposition (HHD). This decomposition describes the flow as the sum of rotation-free, divergentfree and harmonic vector fields. Considering a smooth vector field as the result of the optimally interpolated fields, we can guess the fields associated to the divergence (also known as rotation-free), together with the flow associated to the vorticity which is divergent-free. More details and explanations are provided in Bhatia et al. [6]

\section{Result And Discussion}

The oceanic surface current was calculated during the high tide period, i.e., from the $17^{t h}$ to the $20^{t h}$ of October, 2020. The resulting interpolated map resolves one-hour time intervals on a spatial grid of $1 / 24^{\circ}$. An example of the retrieved total oceanic surface current is shown in figure 5 (a). The Atlantic Jet is clearly distinguished in the eastern part. Although in the western part of the strait the current is opposite and corresponds to the tidal current at the end of the outflow. (The period of inflow corresponds to the eastward current, in the same direction as the Atlantic Jet). In such a case as this, the divergence provides a precious tool to analyse the vertical motion of the ocean. The divergence and the associated rotational-free flow field for the same date are plotted in figure 5 (b). In the region located near longitude $5.7^{\circ} \mathrm{W}$, a high positive divergence is present in close proximity to a negative one, located slightly to the east. This geophysical signature corresponds exactly to the presence of the Camarinal Sill, illustrated in figure 1 (b).
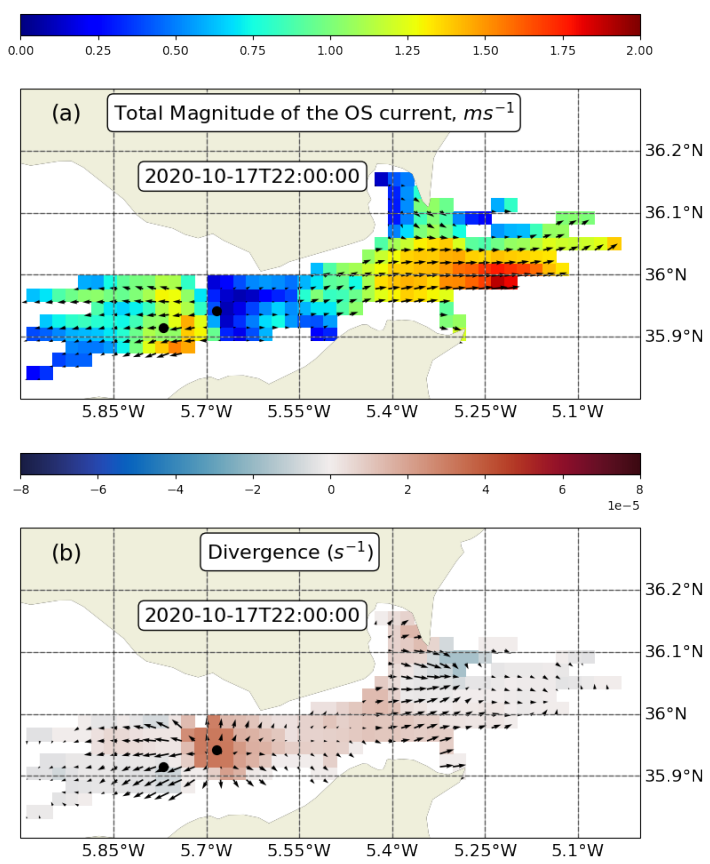

Fig. 5. (a) Map of the total ocean surface current for October $17^{\text {th }}$ at 22:00. (b) Divergence and associated rotational-free flow field obtained through the HHD decomposition at the same date and time. 
The results of the analysis of the time-series concerning the zonal and meridional components on both sides of the Camarinal sill are shown in figure 6 . For the zonal component, shown in plot (a), we note a very marked semi-diurnal oscillation. The two high-amplitude signals are correlated, ranging from $\approx-1.5 \mathrm{~m}$ to $\approx 1.5 \mathrm{~m}$. Regarding the meridional component, shown in plot (b), the amplitude is much lower, ranging from $\approx-0.25 \mathrm{~m}$ to $\approx 0.5 \mathrm{~m}$. The correlation and the semi-diurnal signal are much less pronounced. The most interesting result is obtained from the divergence estimates on both sides of the sill, shown in plot (c). Indeed, in the outflow situation, when the zonal component is negative, the divergence east of the sill is positive, whilst negative to the west. A similar but opposite phenomenon is observed in the inflow situation, i.e., the zonal component is positive and the divergence east of the sill is negative, whilst positive to the west. Our results demonstrate a clear negative correlation and reveal the vertical motion of the ocean due to the interaction of the tidal current with the sill but also the presence of the hydraulic jump to the west of the sill. This phenomenon is related to the formation of the Internal-Solitary Wave (ISW) [7].

\section{CONCLUSION}

In the present work, we exploited the significant volume of AIS data messages to produce fields of the total oceanic current at a depth corresponding to vessel draught $(\approx 10 \mathrm{~m})$. The High Frequency radar located to the west of the area measures the oceanic current just below the surface $(\approx 10 \mathrm{~cm})$. In future work, special attention will be addressed to the comparison of these different sources of current measurements. A clear benefit of using AIS message data is the opportunity it provides for calculating the current fields beyond the HF radar coverage, and more particularly around the Camarinal Sill, site of the ISW formation. The intensity of maritime traffic in this region provides a sufficient AIS data sampling rate to detect the impact of the ISW on vessel speed. In future work, further development of this method will be dedicated to spacio-temporal colocalisation of the formation of the ISW based on AIS data.

\section{ACKNOWLEDGMENTS}

The authors acknowledge that the French Defense Procurement Agency (Délégation Générale pour l'Armement) funded the campaign reported here through Protevs II, an upstream research program (Programme d'étude amont, DGA). We are thankful to the technical team at the French Navy's Hydrological and Oceanographic Service (SHOM) as well as the crew of the R/V L'Atalante for their contribution to the field experiments.

\section{REFERENCES}

[1] J. Soto-Navarro, P. Lorente, E. Álvarez Fanjul, J. Carlos Sánchez-Garrido, and J. García-Lafuente, "Surface circulation at the strait of gibraltar: A combined hf radar and high resolution model study," Journal of Geophysical Research: Oceans, vol. 121, no. 3, pp. 2016-2034, 2016. [Online]. Available: https://agupubs.onlinelibrary.wiley.com/doi/abs/10.1002/2015JC011354
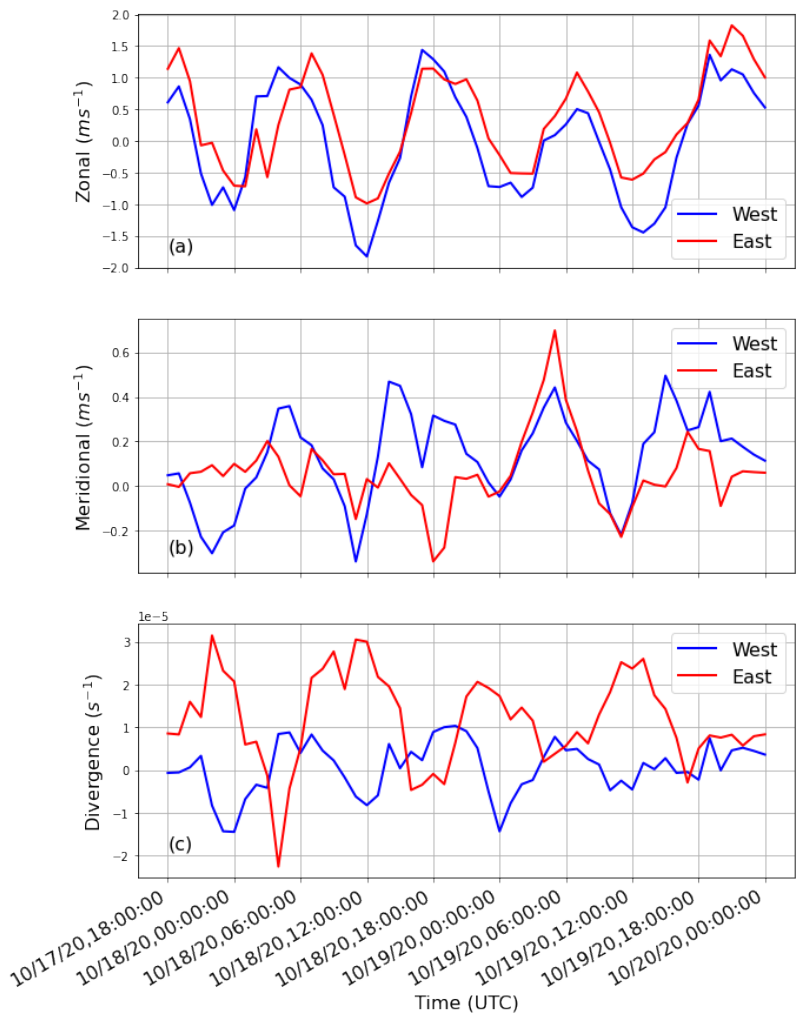

Fig. 6. (a) Time-series of the zonal component of the oceanic surface current at points located to the east and west of the Camarinal Sill (red and blue respectively). Locations are shown in figures 1 and 5. (b) Same as (a) but for the meridional component. (c) Time-series of the divergence of the interpolated field at the same locations.

[2] P. L. Richardson and T. K. McKee, "Average seasonal variation of the atlantic equatorial currents from historical ship drifts," Journal of Physical Oceanography, vol. 14, no. 7, pp. 1226-1238, 1984. [Online]. Available: https://doi.org/10.1175/1520-0485(1984)014;1226:ASVOTA 2 2.0.CO;2

[3] P. L. Richardson, "Drifting in the wind: leeway error in shipdrift data," Deep Sea Research Part I: Oceanographic Research Papers, vol. 44, no. 11, pp. 1877 - 1903, 1997. [Online]. Available: http://www.sciencedirect.com/science/article/pii/S0967063797000599

[4] N. E. Huang, Z. Shen, S. R. Long, M. C. Wu, H. H. Shih, Q. Zheng, N.-C. Yen, C. C. Tung, and H. H. Liu, "The empirical mode decomposition and the hilbert spectrum for nonlinear and non-stationary time series analysis," Proceedings of the Royal Society of London. Series A: Mathematical, Physical and Engineering Sciences, vol. 454, no. 1971, pp. 903-995, 1998. [Online]. Available: https://royalsocietypublishing.org/doi/abs/10.1098/rspa.1998.0193

[5] F. P. Bretherton, R. E. Davis, and C. Fandry, "A technique for objective analysis and design of oceanographic experiments applied to mode-73," in Deep Sea Research and Oceanographic Abstracts, vol. 23, no. 7. Elsevier, 1976, pp. 559-582.

[6] H. Bhatia, V. Pascucci, and P. Bremer, "The natural helmholtz-hodge decomposition for open-boundary flow analysis," IEEE Transactions on Visualization and Computer Graphics, vol. 20, no. 11, pp. 1566-1578, 2014.

[7] D. M. Farmer, L. Armi, L. Armi, and D. M. Farmer, "The flow of atlantic water through the strait of gibraltar," Progress in Oceanography, vol. 21, no. 1, pp. 1 - 103, 1988. [Online]. Available: http://www.sciencedirect.com/science/article/pii/0079661188900559 